\title{
Design and Implementation of Herbal Therapy Knowledge Management System (HTKMS)
}

\author{
Oladejo Bola Fausat ${ }^{1}$, Theodorio Adedayo Olayinka ${ }^{2}$ \\ ${ }^{1}$ Department of Computer Science, University of Ibadan, Ibadan, Nigeria \\ ${ }^{2}$ Departement of Computer Science, Oyo State College of Agriculture and Technology, Igboora, Nigeria
}

Email address:

oladejobola2002@gmail.com (O. B. Fausat), theodoriodayor $@$ yahoo.com (T. A. Olayinka)

\section{To cite this article:}

Oladejo Bola Fausat, Theodorio Adedayo Olayinka. Design and Implementation of Herbal Therapy Knowledge Management System (HTKMS). Science Journal of Public Health. Vol. 7, No. 2, 2019, pp. 44-52. doi: 10.11648/j.sjph.20190702.13

Received: February 21, 2019; Accepted: April 4, 2019; Published: May 15, 2019

\begin{abstract}
The recent influx of herbal mixtures in Nigeria today is gradually becoming alarming. It remains a major concern in the society especially if the right approach is used. Experts believe that the knowledge on herbal therapy is usually obtained from an implicit knowledge of an expert which is transcribed to document-based for reusable purposes. Nowadays, lack of a collaborative herbal therapy system and a massive introduction of different kinds of herbal mixtures in the market today remain a concern for deliberation. This study was designed as a result of lack of communicable herbal therapy platform in Nigeria and secondly, self-imposed solutions to health challenges. The concept of collaborative, user-friendly problem solving software was designed for the study, a package named Herbal Therapy Knowledge Management System (HTKMS). In this study, herbal experts provide expert analysis and solutions to ailments (problems) using the formidable approach of Case-Based Reasoning Model. Case forms, questionnaire and interview were used to gather information from herbal experts and users. Knowledge on Ailments causes and solutions gathered were represented using Entity Relationship Diagram. Case-Based Reasoning technique was adopted for information retrieval from herbal corporate memory (Case Forms) while cases not found were redirected to a collaborative forum for further discussion by herbal experts. Communication among the users (patients) and herbal experts were based on the principles of server-client network. The system was coded using PHP, HTML and CSS. The results of quantitative analysis of the questionnaire developed for the study showed that herbal experts strongly agreed that they diagnose patients manually (Mean Score: 1.90; Std Dev: 0.657), that they proffer solutions from personal experience and presumption (Mean Score 2.90; Std Dev: 0.852), they strongly agreed that they often do not seek for information from practicing colleagues or from a media (Mean Score: 1.85; Std Dev: 0.671) and lastly, they depended naturally on roots, stems, leaves and shrubs for herbal solutions as contained in their "account book" (Mean Score: 1.85; Std Dev: 0.745). Conclusively, the study was able to produce collaborative and reusable software which focused majorly on producing a more organized and reusable herbal knowledge for users.
\end{abstract}

Keywords: Case-Based Reasoning, Experts, Users, Server-Client Network, Ailments and Knowledge

\section{Introduction}

Knowledge is classified into five meanings: awareness of facts and solutions; a state of being informed; products of learning; a piece of information and familiarity (understanding of a particular skill) [1]. Knowledge application as cognitive processes to information can be developed and applied having being formed by human beings. Knowledge as an information is interpreted within a context by human beings, a perception about a fact or an event in certain contexts [2]. This assertion corroborates the belief of Oladejo and Osofisan on what the definition of knowledge should be [3].

Oladejo and Osofisan believe that first, knowledge creation is from information acquired and secondly, a context. The Who, What, Where and When questions must be answered. Thirdly, knowledge created of acquired must be stored up for reusable purposes; this is often referred to Knowledge Management System (KMS). Knowledge Management System is a process that allows resources to be managed in order to facilitate access and reuse as seen in the five stages of knowledge management system: 
identification, capturing, representation, storage, sharing, use and reuse. Knowledge Management System contains facts gotten from an old repository system and later used up to form an interactive repository system which are in most cases, online. Knowledge Management System has so many applications with respect to area of discipline but for the purpose of this study, it is limited to computer science. KMS as a computer program reasons and uses knowledge base to solve complex programs, Knowledge management system turns information required through its tools into knowledge acquired. Knowledge management system is distinct but interdependent process of knowledge creation, knowledge storage and retrieval and knowledge application [3-4]. Knowledge is a fluid mix of fumed experiences, values, contextual information and experts' insight that provide a framework for evaluating and incorporating new experience and information [5]. The view of experts on KMS is such that knowledge originates from the individual and is used by an individual's mind thus helping to solve a problem, enhance performance, integrate learning and teaching through idea sharing, personal experience, intuitions, skill generation and values [6]. The explanation given corroborates the areas of context and problem solving techniques; a scenarios that describes knowledge creation process for human use [2, 5]. This definition on KMS could be interpreted in such a way that for all knowledge created, it must be for human use and should be applicable to sciences, organizations or societies. Organizations make resilient efforts in searching for information that could improve their productivity, an act that often leads to acquisition of facts and figures.

Recent influx of herbal remedies despite the availability of orthodox medicine into Nigeria is becoming alarming; the genuineness and usefulness of all these remedies may not be ascertained except if an insightful analysis is done. As seen in orthodox medicine where drug certification is done via short messages and regulations, herbal practice is not the same. Orthodox medical experts do relate online through a responsive collaborative forum whether online or offline in which case history of ailments is analyzed, herbal practice does not follow the same practice. Imagine a scenario where "herbal doctors live in far distant places and do need help on unidentified ailments. The need for information on herbs for ailment as well as methods of preparation remains a missing link in Nigeria herbal system". Problem such as the lack of a platform for information sharing by herbal therapists remain a major disturbing issue in the scenario described above, a concern that gave birth to this study, its aim is to develop a knowledge management system for herbal therapy, a case study of selected herbal homes in Nigeria with emphasis on sharing and collaboration. However, its objectives include: to identify and capture ailments and their herbal solutions from herbal experts, to create a collaborative forum for discussion by herbal experts and to create a Herbal Therapy Knowledge Management System for reuse purposes.

\subsection{Review of Related Works}

Herbal medicine is the use of herbs to treat sicknesses or the use of herb for therapeutic and medicinal values [78]. Herbal medicine has a strong relationship with the society today, evidence shows the availability of green leaves and plant and an easy access to these plants even in the creation of the universe. The scripture revealed that God gave man herbs, trees, fruits and seeds for use[9]. Conjuncting these arguments herbal therapy is an end to a means, the means being the hospital [7]. It was argued that colonial education did more harm to corrupt the thinking of Africans [10]. Isaac further described herbal medicine as botanical medicine or phyto-medicine as defined by Maryland medical center as the use of plant seeds, berries, roots, leaves or flowers for medicinal purposes. These plants have been used from time memorial. For example, in Nigeria, it is majorly in use before colonization, and even after colonization, it is proctored as ever, though with new inventions and fabrications.

The use of herbs in Nigeria started way back before colonialism, a situation when there was no availability of orthodox medicine up to the days of orthodox medicine because drugs made from orthodox medicine were actually refined from leaves, stems, roots, and shrub. Parents in these early days of creation, mixed special plants like lemon grass, cashew trees, lemon trees, lemon tree and the likes for fever. These trees had been in use for years before civilization and even at this, the use continues to extend spread across all geo political zones in Nigeria. A study reported that traditional medicine had existed in Nigeria prior to era of colonialism and serving the health needs of the people since all the societies desired good health for their members [11]. Nigeria tribune reported that $75 \%$ percent of Nigerian population still prefers to solve their health problems by consulting traditional healers [11-12]. A study further used ontology for knowledge representation of Africa Traditional Medicine (ATM) especially in the South-West Nigeria [13-14]. The objective of the study was to build an ontology model through formalization of logic representation. A logic based inference, facet coupled with DL rule of inference and ontology knowledge based editor were used in their approach of knowledge representation.

The study recommended a web based ATM software to grant access to information. This web based ATM will help ease down psychological consideration and trauma experienced by practitioners of ATM. Furthermore, a logic based inference engine will reason and make decision over the ontology. The author recommended an extension into collaboration stage of Knowledge Management System [14].

A researcher illustrated traditional medicine (TM) and how its knowledge is generated as a comparative analysis of locally made herbs [15]. Countries like United States of America, India, China and Zimbabwe were used for the 
study. Cases like misuse, safety, documentation, database and code of conducts were examined. For instance, magic medicine in Zimbabwe was considered to be supernatural. Identification of these herbs is through a system that identifies diagnoses, prepares and documents herbs products and treatments history. An ephedra sinica in U.S.A was examined. It was established that ephedrasinica is an old Chinese herbal pharmacopeia which has high concentration of toxic reaction which may lead to heart attack and stroke. The authors acknowledged the functionality of a functioning Traditional Knowledge Digital Library (TKDL) as practiced in Asian Countries like India. The objective of TKDL is to compile and widely disseminate information on Ayurveda (a traditional herb in India and Asian countries). Moreso, the use of documentation of TMK (Traditional Medicine Knowledge) helps preserve knowledge for future use. It provides a vital step in facilitating research on TM. Documentation assists in obtaining information on certain types of IP (Internet Protocol) protection which may help TMK holders to have basic research on healing properties of medicinal plants.

Additionally, the authors discussed the importance of TKD (Traditional Knowledge Databases). Their research shows that TKBD exists in different forms: symptoms, materials, treatments e.t.c. TKDB refers to compilation of traditional knowledge (Traditional Knowledge Database). This knowledge may be printed or electronically stored. Electronic database are simple and easy to access (sometimes protected under the law).

The importance of herbs is not limited to medicinal benefits but to local and traditional herbs. It was observed that knowledge of traditional herbs is unstructured and scattered [16]. A web ontology language using Protégé 4.2 was proposed for knowledge representation and implementation using Malaysian indigenous herbs [16]. Ethologically, the authors used interview sessions to extract information from herb experts. The validated information was prototyped using ontology classes which later converts the OWL (Web Ontology Language) into a database system. In their methodology, four steps were taken:

i. They enumerated all terms in herb domain

ii. They defined terms

iii. They defined class and hierarchy

iv. They identified relationship among classes.

The result of the study stated that in the preparation of herbs, six classes of preparation were identified: decoction, drying, extraction, direct usage, peel and lastly, incision. From these findings, unstructured information became structured and organized. It was discovered that classes of plants used has capability to cure ailments by using some of the plants or part from categories like roots, stems, barks, fruits or whole plant. A model was further developed using subclasses (shrub, tree, e. t. c.) to herb species to leaves, stems, roots e.t.c. and to usage (direct, decoction, peel e.t.c.). The model also highlights the preparation method from the indigenous herb. Furthermore, an entity-relationship model showing identification, diagnosis, and treatment of identified ailment was developed to further simplify understanding of the model proposed as TMM ontology. It was concluded that information on ailment can be captured with the use of interview and represented ontologically with OWL2DB (Web Ontology Language Database) algorithm.

A representation of knowledge using ontology as a formal explicit description of concepts in a domain course is often called classes, each having properties that will describe various features and attributes of concepts [17]. The ideology was the use algorithm for data mining (retrieval). One would agree that the place of collaboration in any profession can never be overlooked as it gives room for diverse understanding and updated knowledge. Literature perused above discussed ways of identifying relationships and hierarchies in herbs, much was not said about how to identify solution to new problem through collaboration, a vital stage of knowledge management system. Evaluating the research works, we observed that knowledge of domains were represented well enough but there is still need for collaborative sharing, since stages of Knowledge Management System is never completed if one stage is omitted. Collaboration produces diverse knowledge and experience on pivot issues which creates information sharing: a vital tool of updated knowledge to archaic knowledge.

\subsection{Methodology and Design}

The study adopted the six stages of knowledge management system development stages: information gathering, capturing, representation, storage, retrieval and reuse. Interview sessions and case forms were designed for easy identification of ailment as well as solutions. Furthermore, an entity-relationship diagram and class diagram were used to show relationships respectively.

A central memory known as Herbal Therapy Knowledge Management System (HTKMS) Corporate Memory was created for storage of cases. This HTKMS Memory consisted of solutions from history (experience) and solutions from collaborative forum. A Case-Based Reasoning (CBR) was adopted for retrieval of solutions from history of cases. The contents in the CBR came from case forms filled by users (patients), herbal experts and interview conducted. A case not found in the CBR (solution) was directed to a collaborative forum for further discussion by herbal experts. Figure 1 below shows the technicalities involved in HTKMS designed. The architecture describes the Who, What and how a solution is arrived at for identified ailments. The 'Who' includes herbal expert and a Patient (Users) in question? These two set of people are the major stakeholders in the study. A patient can be a user and can also be an herbal expert. The means of identification is through registration. The registration interface helps to identify who is retrieving from the system or who is contributing from experience. The 
second phase is the therapy phase. This phase can only be used by herbal experts alone. They contribute by sharing the sign and symptoms seen for identified ailment and the solution used for the identified ailment. Also, they discuss the procedure for creating solution and also, the measurement.
Another form of solution is the solution from the collaborative forum. Here, experts freely give their opinions on identified ailments as well the solvable solution. These solutions are stored in a single memory called HTKMS Corporate Memory.

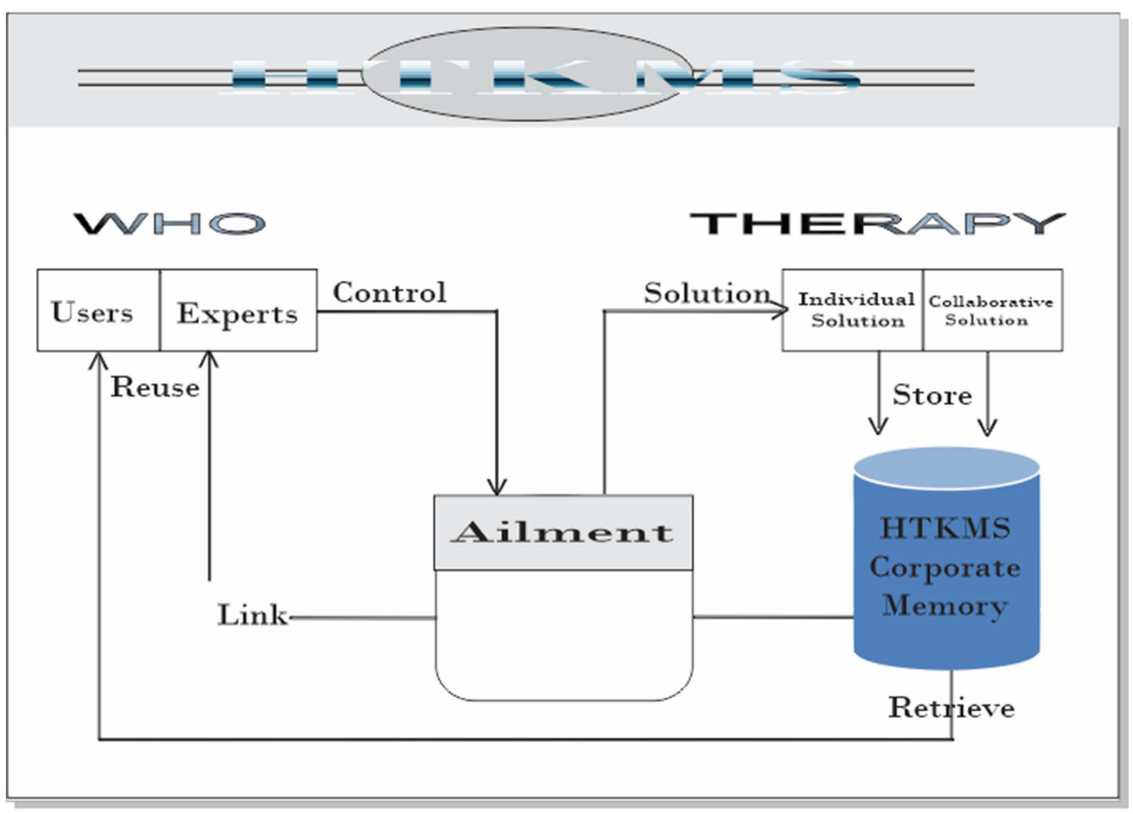

Figure 1. Architectural Design for HTKMS.

Retrieval from the memory is done through the use of Case-Based Reasoning Technique. According to [21] Choy (2003), Case-Based Reasoning Technique fetches solutions to a problem from history of similar problems stored in a memory. The case based reasoning is useful for inferential analysis of cases and a referral to similar problems as no problem exists without a history. [22] Selmer et al, (2003) defined content based reasoning as a philosophy that combines knowledge-based support system with a simulation of human reasoning of past experience. An idea of using explicit past documentary evidences to solve problems, usually done with case files and documents. These case files or documents are real life experiences.

The Use-Case diagram was used to model the interactions between users and herbal experts in the designed systemHTKMS as shown in figure 2 below. A user or herbal expert must register as stated earlier, the difference in user is such that a user/patient can only make enquiry or retrieve from the corporate memory while an herbal expert will proffer solution through the collaborative forum. The forum allows herbal experts to update their knowledge, contribute from personal experience or encounter. The solution or comment given by herbal experts together with case notes gotten from herbal experts make up the corporate memory

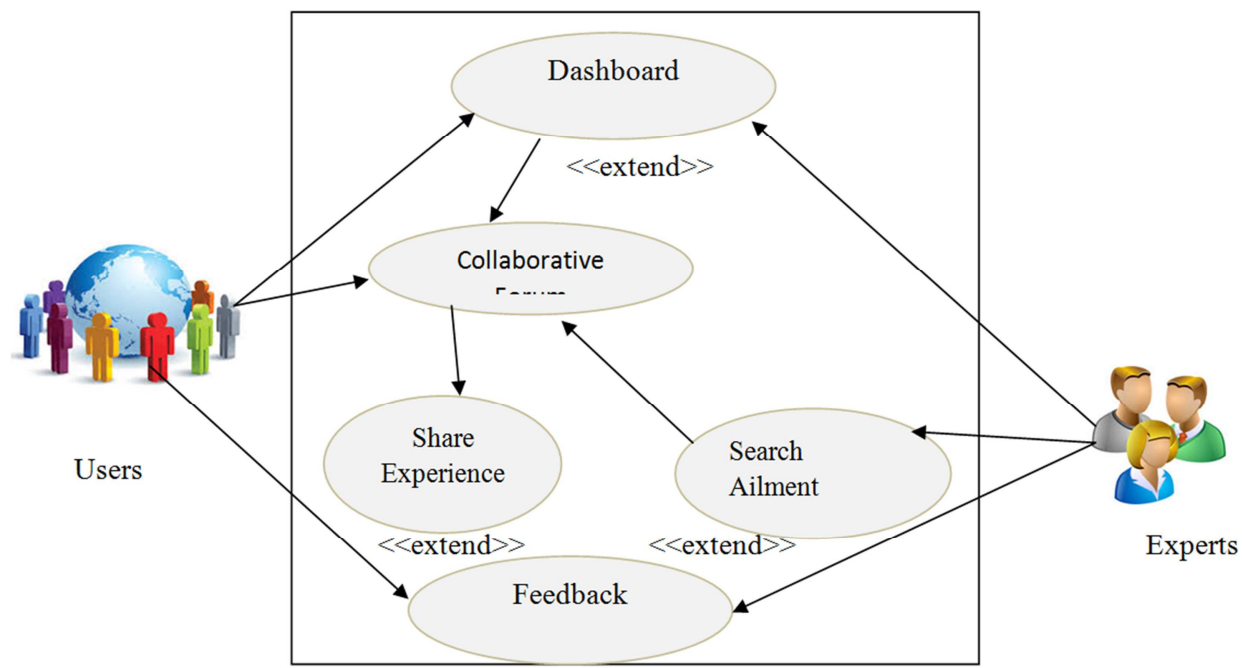

Figure 2. Use-Case Interaction Diagram for HTKMS. 
An Entity-Relationship (E-R) diagram was used to show relationship between personal messages/opinions on topics. Topics (ailment) were grouped into categories. The collaborative forum was developed for herbal experts (users). It enabled experts' criticism, arguments as well as suggestions on ailment identified and possible solution(s). The communication transmission protocol used for the study was clients-server transmission protocol. Clients necessitate solutions from herbal experts if cases are not found in the case-based memory. The herbal experts then pick up new cases for discussion and expert analysis of the identified problem. The designed system works in such a way that "not found cases are pushed into the collaborative forum. Herbal experts gain entry through validation of credentials". It is to be noted that only herbal experts can participate in the forum, this is determined through the use of group concession on solution to new problems.

The process of recommending solutions to users or patients was made possible with the principles of serverclient network protocol. A server-client network adopted because the system involved many users and a centrally controlling server- the administrating computer that populates and control inflow of information. Client computers are differently placed users (patients) who needs information on health issues and if herbal solution will be suitable. The deployment of the system designed followed the algorithm written below:

Step 1: Login

Step 2: Users (patients) input ailment/problem

Step 3: Retrieve solution from Case-Base

Step 4: Not seen? Push into Herbal Therapy collaborative forum for expert discussion
Step 5: Expert enter registration details

Step 6: Comment on ailment submitted into Case-Base, Logout

Step 7: Updated solution saved in corporate memory

Step 8: Users (Patients) fetch updated solution

Step 9: Users Log out.

Figure 3 below shows the operational procedure of the system HTKMS. An ailment would be searched for in the corporate memory; a decision making on solution queried was enabled to ascertain if the solution is found or else it will be redirected to the collaborative forum. If found, the user accesses the solution and if not the needed solution, a user is free to submit a form for experts' view and analysis.

In the collaborative forum, every comment/recommendation is validated via group concession. Only a comment with higher votes or positivity will be populated by the administrator. Furthermore, a user complaint form is filled for every not found case while an herbal expert form is filled for populated new case(s). The user form contained information of patients as well as test status and ailment confirmation while the herbal expert form contained experts' information, previous experience on the ailment, causes and solution.

The communication protocol for the system HTKMS followed clients-server architecture as described earlier. Information is released by the server computer which controlled the flow of communication. It also supported feedback for all client computers. In this study, client computers were users (patients) while the server computer (herbal expert) was a centralized computer that monitored the relevance of what is populated into the HTKMS Corporate Memory.

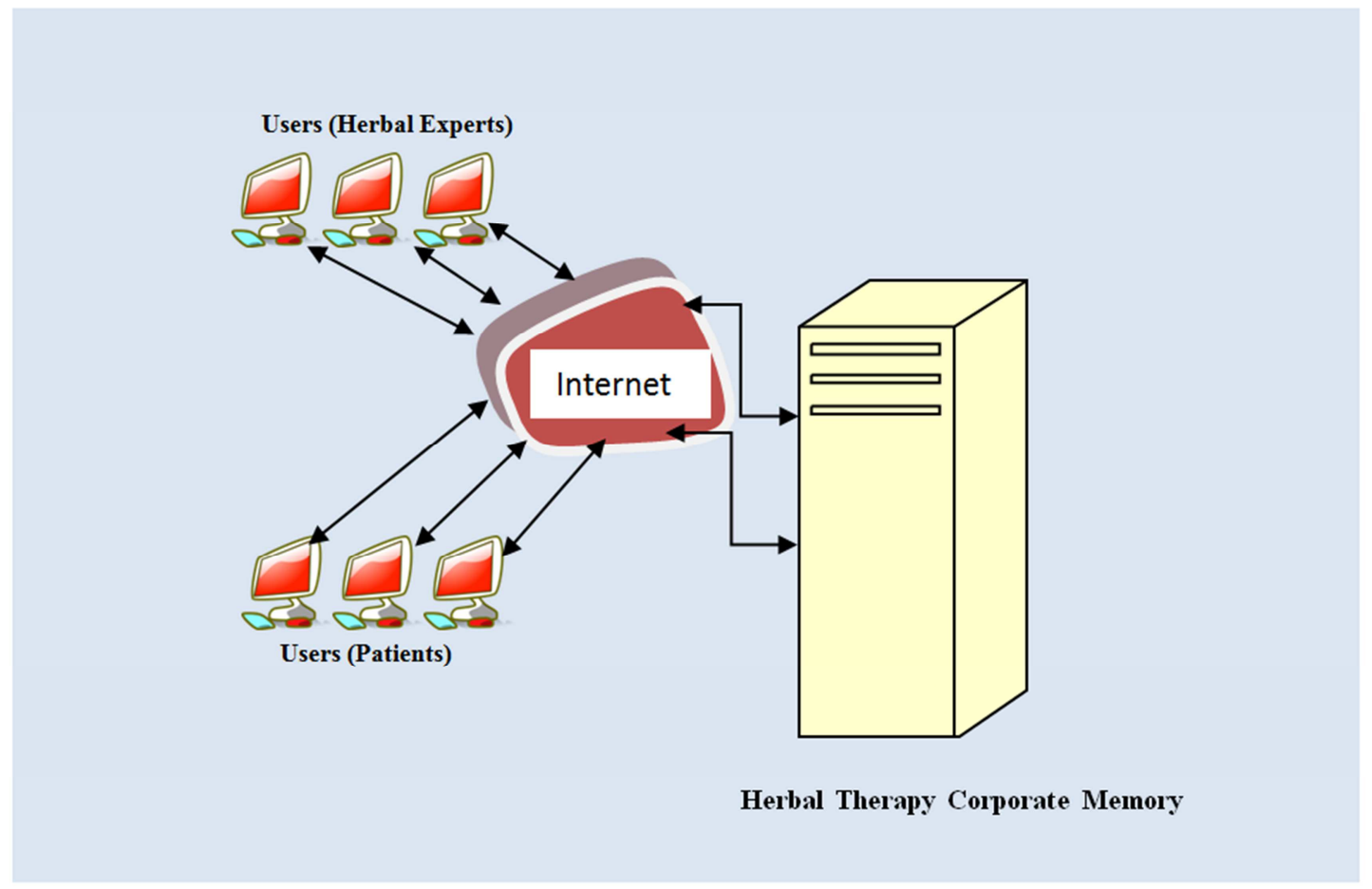

Figure 3. Users Communication Process. 
The registration interface allows the system to capture records of all users: both users and herbal experts. Records of users are stored in the corporate memory in order to link comments and solutions provided.

\subsection{Statistical Results}

The study used interview method and questionnaire for information gathering. Herbal homes were selected randomly based on accessibility and availability of herbal experts. A total of twenty homes were selected from Lagos State and Oyo State. The questionnaire was designed with a 4-likert scale format (A-Agree: 1, SA-Strongly Agree: 2, D-Disagree:3 and SD-Strongly Disagree:4). It was divided into three sections: Section A for demographic details, Section B was divided into A and B. The A part was used to gather information on target problem identification while the B part was used for solution identification. Section C contained glossary of ailment, causes, symptoms and herbal solution. The interview sessions conducted concentrated on technology use for problem identification, experience, collaboration with other experts and willingness to adopt the new herbal platform. The result of the questionnaire indicated for herbal solutions as contained in their "account book" (Mean Score: 1.85; Std Dev: 0.745), herbal experts strongly agreed that they diagnose patients manually (Mean Score: 1.90; Std Dev: 0.657), they strongly agreed that they proffer solutions from personal experience and presumption (Mean Score 2.90; Std Dev: 0.852), they strongly agreed that they often do not seek for information from practicing colleagues or from a media source (Mean Score: 1.85; Std Dev: 0.671). Lastly, they depended naturally on roots, stems, leaves and shrubs (mean: 1.85, std dev: 0.745). Extract from the interview sessions conducted revealed that experience played a major role in the practice of herbal medicine as majority of the herbal experts had between ten to twenty years' experience on the job. They agreed that they are willing to adopt the software if they are properly trained and supervised as it will further make their practice popular and accessible. However, majority of them do not engage in discussion or consultation with other experts or any source outside their inherited source of information. Furthermore, they depend solely on divine consultation (except for One Mr. Owoade Johnson who practices Ortho-Herbal Medicine).

\subsection{System Outputs}

The outputs of the system designed are presented below:

\subsubsection{Input Stage}

The registration page was designed to capture details of users especially their name, chat-name, username, password, email address and date of birth. With all these, record of activities of users was monitored. Furthermore, record of ailment was populated using three approaches as discussed: the user case form, the expert case form and case note collected during interview sessions (fact finding). The user case note emanated from "case not found situations".

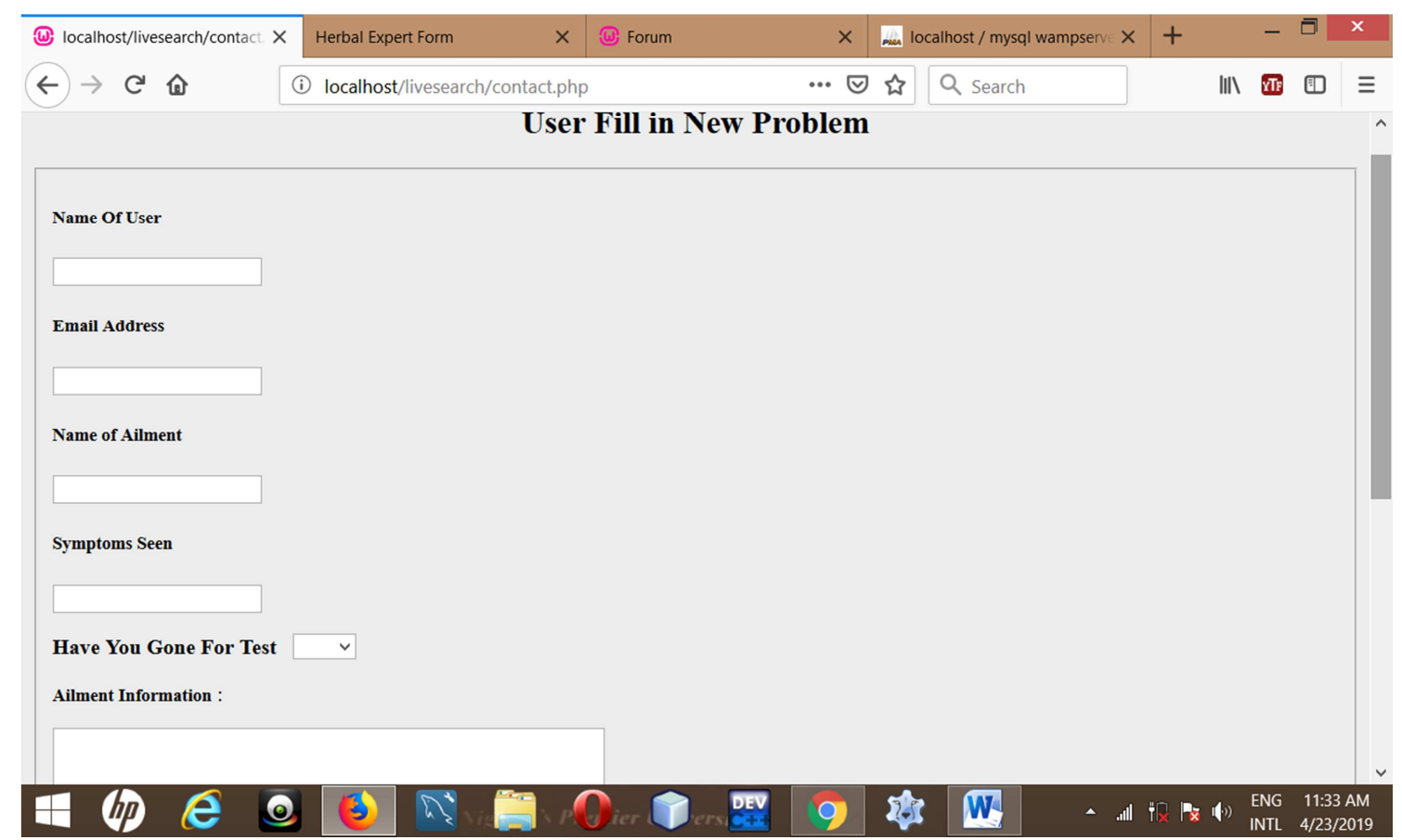

Figure 4. HTKMS Input Stage.

These case forms will be populated to form new case notes. The users' end will only populate while the herbal experts contribute solution through collaboration of personal experience. The HTKMS has two interfaces in one system: the user end and the Herbal Expert end. 


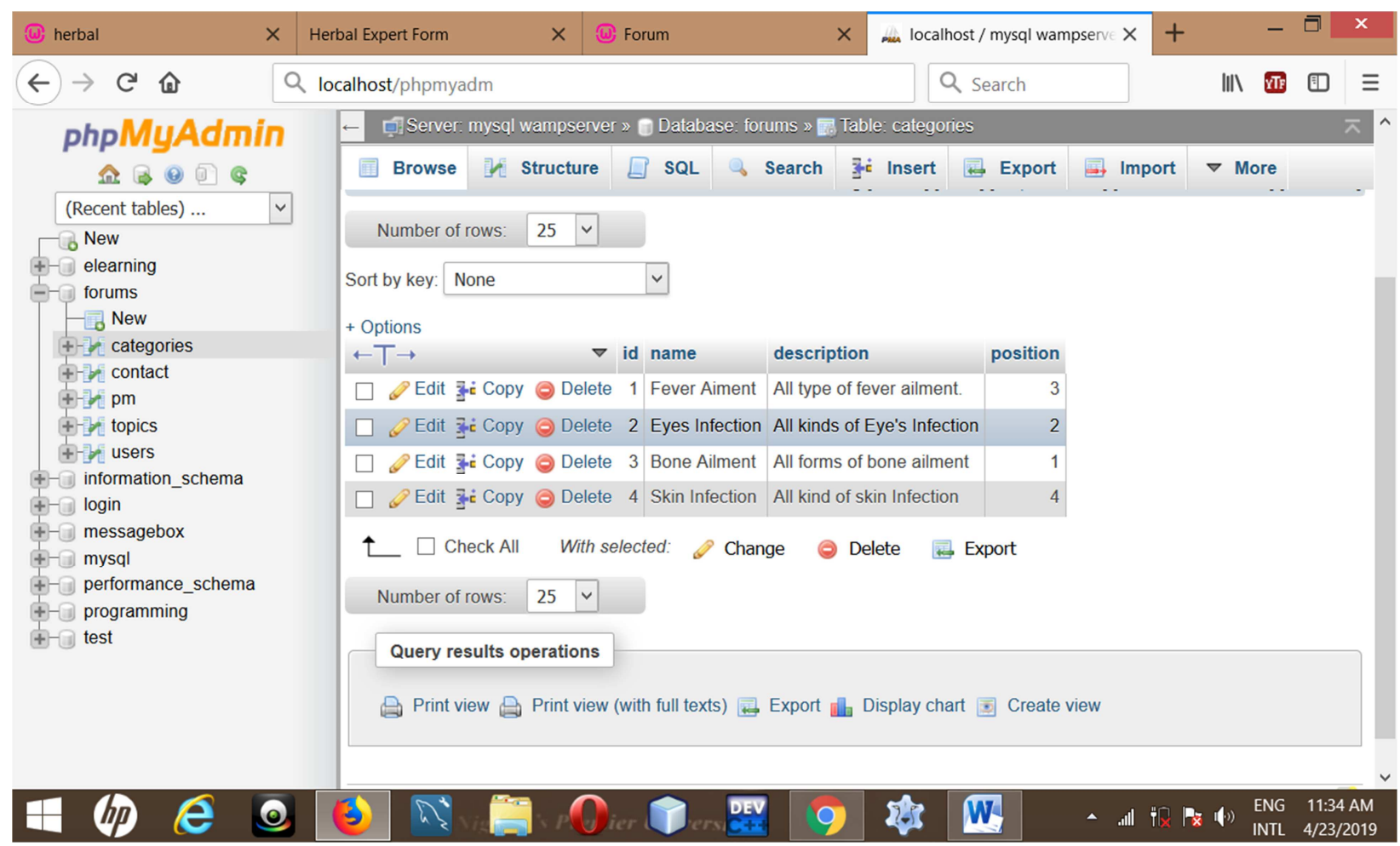

Figure 5. Populated Cases in a Database.

\subsubsection{Storage Stage}

Contribution at the herbal expert end can be done in two ways: either through collaborative forum or through filling of herbal expert form. A group concession form was filled by a certified herbal expert before it is populated.

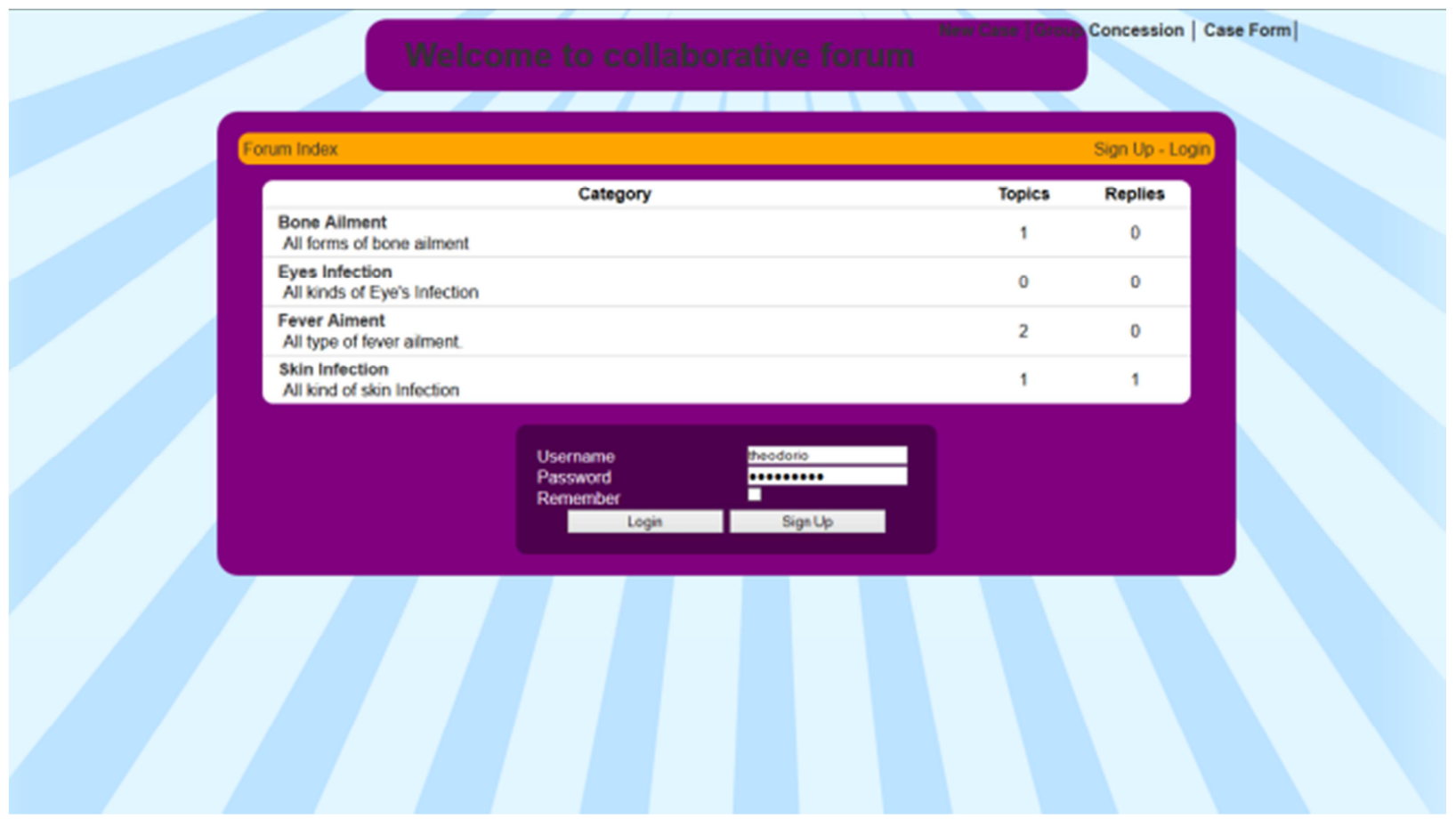

Figure 6. Collaborative Forum.

\subsubsection{Retrieval Stage}

The result of the design clearly show how knowledge of herbal medicine can be managed using the best practices of Knowledge Management System. When all these information are gathered, the HTKMS was populated for feedback. 


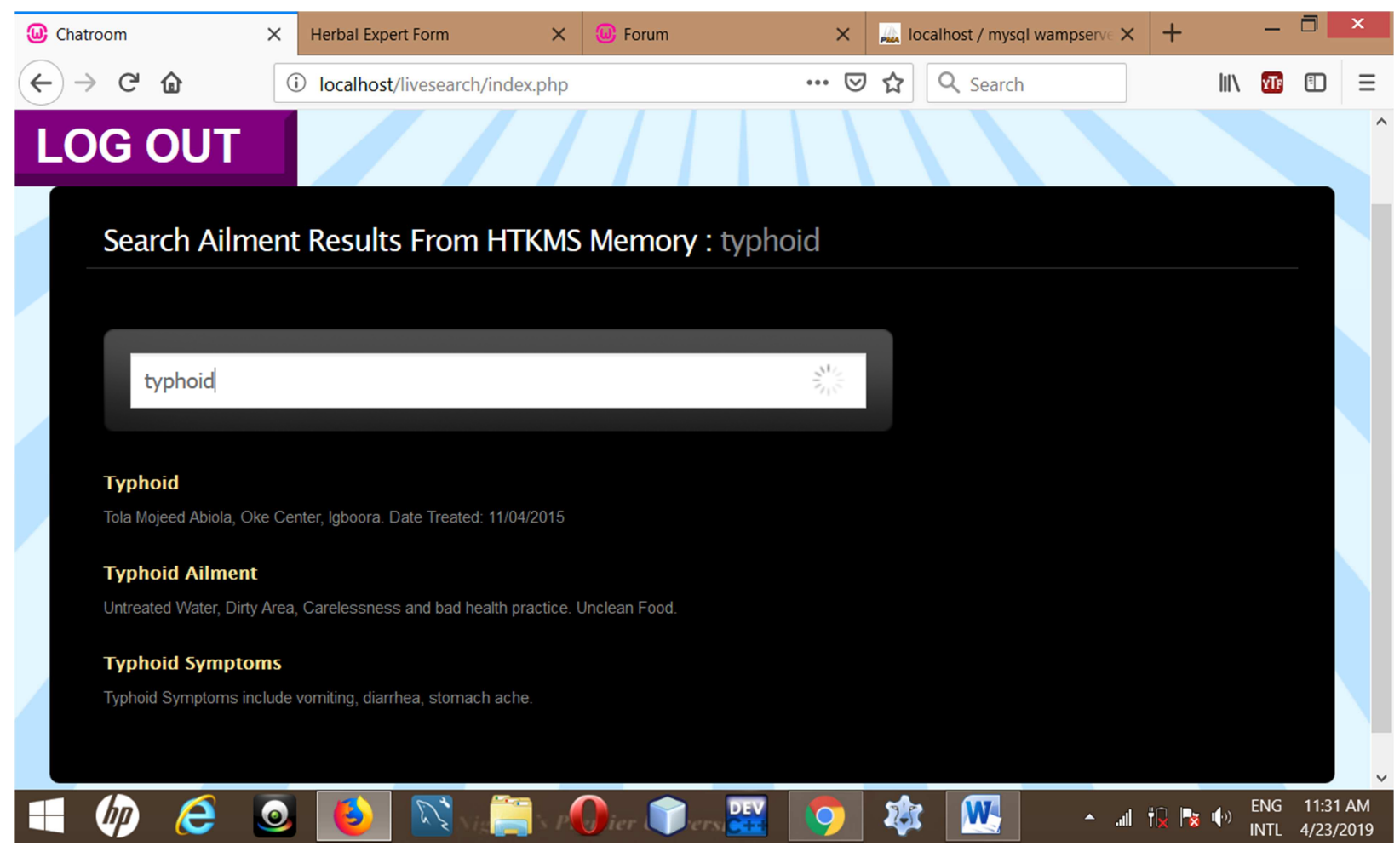

Figure 7. HTKMS Populated Memory.

\section{Evaluation}

The system was evaluated by observing methods of knowledge representation adopted by authors concerned. Technically, majority of these studies focused on knowledge acquisition and representation, majority left out the issue of collaboration. This work was basically a novel research that focused on how knowledge can be created, represented and stored. The knowledge case-base method of knowledge repository was critically studied and adopted for the development of herbal therapy knowledge management system.

\section{Conclusion}

Knowledge of herbal therapy is never customized and never stagnant. This study remains an evolutionary research that investigated how knowledge on herbal therapy and ailment can be represented and re-used through collaboration and information sharing among herbal experts and users. The study made a decent and thorough job in solving the problems identified and to provide solutions through a collaborative efforts by both experts, and users. Herbal Experts were able to share their experience for a wider use. These experience were subjected to validation by other experts too. The retrieval process follows the pattern of Case-Based Reasoning approach for Knowledge Management System. In a case where similar solution is not found, a form would be proffered as available solution process and which will be automatically populated for the experts to ruminate on.

\section{Future Work}

We would work on how to capture ethno-botany constituents of herb e.g. roots, leaves, stems and shrubs of identified trees that could be used as herbal therapy. When captured, we will represent and arrange their uses according to the principles of knowledge management system. This new approach would lead to the recognition of herbal patterns in Nigeria.

\section{References}

[1] Oxford English Dictionary. (2015). English 1. 2. 2 Mobile Application, Android Version.

[2] Oladejo, O. B. and Osofisan, A. O. (2011). A conceptual framework for knowledge integration in the context of decision making progress. African Journal of Computing and ICT. Vol. 4. No. 2. Issue 2. pp. 25-32.

[3] Frank Mckenna. (2008). A knowledge management system. A Discourse. Knowledge One Corporation.

[4] Alavi, M. and Leidner, D. (2001). Knowledge management and knowledge management system: conceptual foundations and research issues. MIS Quaterly. Vol.25, pp. 107-136.

[5] Davenport, T. H. and Prusak, L. (1998). Working Knowledge: how organizations manage what they know? Boston. Mass Harvard Business School.

[6] Liebowitz and Probst. (2000). Beyond Knowledge Management. What every leader should know? 
[7] Isaac, N. D. (2009). Ghanaian indigenous health practices: the use of herbs. Department of sociology and equity studies, University of Toronto. Master Thesis.

[8] Helpful herbalist (2009). A brief history of Medicine. http://www.helpfullherbalist.com/herbalmedicine.html.

[9] The Living Bible. (1976). Genesis, 1. Verse 29. Tyndale House Publishers. ISBN: 978-978-079-572-6.

[10] Appiah, J. (2006). Akan proverbs as indigenous knowing: relevance for schooling and education. Master Thesis. University of Toronto. University of Toronto website.

[11] Adefolaju Toyin. (2011). The dynamics and changing structure of traditional system in Nigeria. International Journal of Health Research. Vol 4 No. 2, pp. 99-106.

[12] Nigerian Tribune. (2008). March 28, 2008. pp. 2. In Adefolaju Toyin (2011). The dynamics and changing structure of traditional system in Nigeria. International Journal of Health Research. Vol 4. No. 2, pp 99-106.
[13] Adesina, S. K. (2011). Traditional medical care in Nigeria. Retrieved from http://www.onlinegeria.com/traditionalmedicalcarenigeria.co $\mathrm{m} / 2011$. 15th November, 2015.

[14] Omotosho, L. O., Odejobi, O. A. and Akanbi, C. O. (2006). A framework for knowledge-based ontology model in African Traditional Medicine. African Journal of Computing and ICT. Vol. 6. No. 1. pp 43-48.

[15] Ryan Abott., M. T. O. M., J. D. and M. D. (2014). Documenting traditional medical knowledge. World Intellectual Property Organization.

[16] Zulazeze, S., Sharifalillah, N. and Haryani, H. (2012). Malaysian indigenous herbs knowledge representation. Knowledge Management International Conference. Johor Bahru, Malaysia. 4-6 $6^{\text {th }}$ July, 2012.

[17] Babajide Olanrewaju. (2013). A knowledge discovery system for news media using text mining techniques. An M.Sc Unpublished Thesis. Department of Computer Science, University of Ibadan. 